\title{
An anatomical study of the origin, structure and insertion of the medial patellofemoral ligament
}

\author{
C.-H. Li' ${ }^{1}{ }^{2}$, D. Ricketts ${ }^{3}$, X.-S. Wang ${ }^{1}$, T.-B. Yu' ${ }^{2}$, C. Qi' ${ }^{2}$, Y.-L. Zhu ${ }^{4}$ \\ ${ }^{1}$ Department of Orthopaedics, People's Hospital of Rizhao Affiliated to Jining Medical University, Rizhao, China \\ 2Department of Orthopaedics, Affiliated Hospital of Qingdao University, Qingdao, China \\ ${ }^{3}$ Department of Trauma and Orthopaedics, Brighton and Sussex University Hospitals NHS Trust, Brighton, United Kingdom \\ ${ }^{4}$ Department of Orthopaedics, Shandong Province Western Hospital, Jinan, China
}

[Received: 11 January 2018; Accepted: 10 March 2018]

\begin{abstract}
Background: Repair and reconstruction of the medial patellofemoral ligament (MPFL) has been undertaken for the treatment of patellar instability. For successful surgery detailed knowledge of the anatomy of the ligament is required. The aim of this study was to describe the origin, structure and insertion of the MPFL.

Materials and methods: We studied cadaveric knees from 30 Chinese adults. We studied the origin, course and insertion of the MPFL.

Results: We found that the MPFL was composed of two main strands, superficial and deep. We also found that the insertion of the MPFL into the femur had three common patterns. All three were located between the adductor tubercle and the proximal part of superficial medial collateral ligament.

Conclusions: We have added to the anatomical knowledge of the structure of the MPFL. Our study has implications for guiding bone tunnel positioning during MPFL reconstruction surgery. (Folia Morphol 2018; 77, 2: 356-361)
\end{abstract}

Key words: medial patellofemoral ligament, anatomy, reconstruction surgery

\section{INTRODUCTION}

In 1957, Kaplan [10] described the medial patellofemoral ligament (MPFL) in a study concerning the stability of the knee. Since then the role of the MPFL in stabilising the patella has been increasingly recognised.

The literature contains varying descriptions of the anatomy of the MPFL $[2,3,5,8,9,13,14,16-20$, 22]. In summary, the MPFL is located medially on the knee between the superficial fascia and the joint capsule. The origin is from the medial patella and vastus medialis tendon. The size of the origin varies widely $[2,18-19]$. The MPFL itself has been described as transverse fibrous tissue [21]. The insertion of the MPFL is also variable and includes the medial epicondyle and adductor tubercle $[3,5,8,9,13,14,16,20,22]$.

This structure is the primary static soft-tissue structure of the medial patellar. It is important in preventing lateral patellar displacement [1, 5]. During acute lateral patellar dislocation the MPFL is often ruptured [15].

Surgical repair and reconstruction of the MPFL is an established method for the treatment of patellar dislocation $[4,6,7,11,12]$. For successful surgery detailed knowledge of the MPFL anatomy is essential. The aim of this study was to describe in detail the anatomy of the MPFL.

\section{MATERIALS AND METHODS}

\section{Specimens}

We studied 30 cadaveric knees. Thirty formalinfixed adult cadaver knee specimens were provided by the Department of Anatomy, Qingdao University, 308 Ningxia Road, Qingdao, China. The experiment was performed with the ethical approval given by 


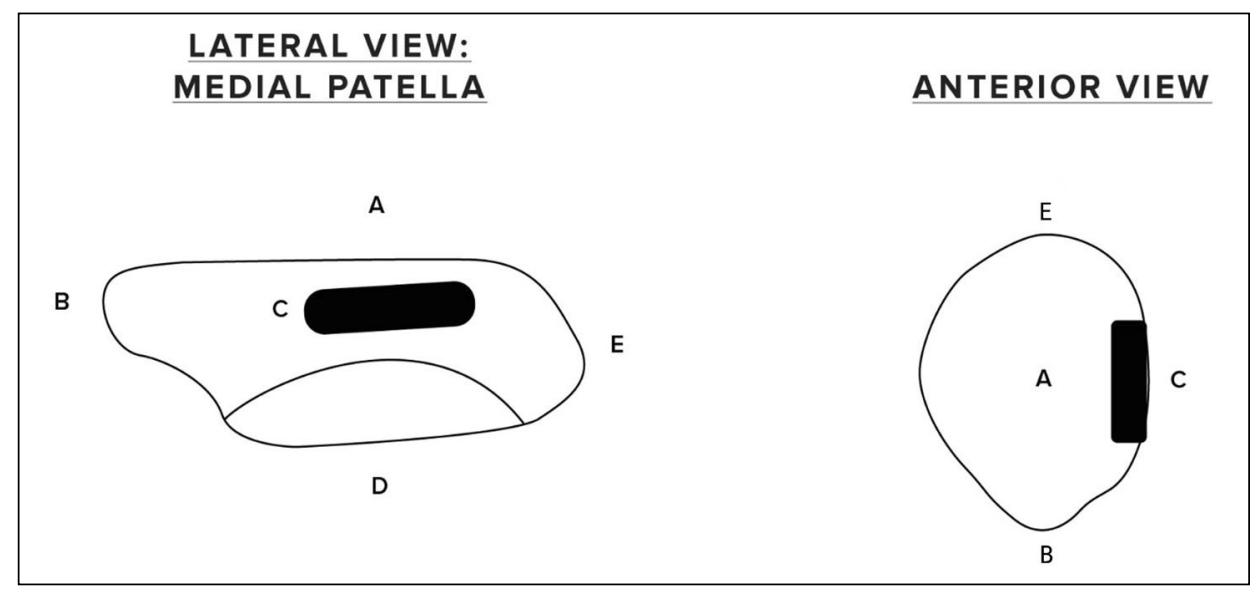

Figure 1. The origin of the medial patellofemoral ligament (MPFL) from the medial patella; $A-$ anterior border of patella; $B-$ inferior margin of patella; $\mathrm{C}$ - origin of MPFL; D - articular surface of patella; $\mathrm{E}$ - superior margin of patella.

the Medical Ethics Committee of People's Hospital of Rizhao with the following reference number: KYLL201703 (Approval Date Jul 1'st, 2017).

The specimens were all from adults, their age at the time of death was $25-45$ years old, the average age was 33 years; 22 were male and 8 were female. We wanted to avoid specimens from individuals with degenerative changes. This might have interfered with the studies' findings. We consequently requested young and middle-aged specimens for this study.

\section{Dissection methods}

The specimens were all dissected by the authors $\mathrm{CHL}$ and CQ. Through a longitudinal incision on the medial side of the knee the medial patellar retinaculum was exposed. We found 5 layers of structures on the medial aspect of the knee. Layer 1: skin and subcutaneous adipose tissue. Layer 2: superficial medial patellar retinaculum. Layer 3: medial patellotibial ligament, vastus medialis oblique aponeurosis, and superficial MPFL. Layer 4: superficial medial collateral ligament, and deep MPFL. Layer 5: medial capsule and medial patellomeniscal ligament.

The MPFL was found by dissecting between the vastus medialis oblique (VMO) aponeurosis and the medial patellotibial ligament. The MPFL extended from the patella to the femoral condyle under the superficial medial retinaculum.

\section{Statistical analysis}

The origin, structure and insertion of the MPFL were carefully observed and recorded. A Vernier calliper (precision of $0.01 \mathrm{~mm}$ ) was used to measure all data regarding the MPFL. Data are presented as mean \pm standard deviation. Data were analysed with SPSS 16.0 statistical software.

\section{RESULTS}

\section{The patellar origin of the MPFL}

The origin of the MPFL was from the medial and distal surface of the suprapatellar tendon, the medial border of the patella and the proximal infrapatellar ligament.

Guided by the direction of the fibres we dissected the MPFL from the suprapatellar tendon and the infrapatellar ligament. This allowed us to identify the patellar origin of MPFL.

The patellar origin of MPFL was from the convex medial margin of the patella. The length of the attachment in the vertical plane was $18.3 \pm 1.1 \mathrm{~mm}$. This was centred on the most prominent medial part of the patella. The depth of the attachment was $1.6 \pm$ $\pm 0.1 \mathrm{~mm}$ (Figs. 1, 2). The thickness of the patella itself in the anteroposterior diameter varied from $10.0 \pm$ $\pm 0.3 \mathrm{~mm}$ at the proximal origin, to $6.2 \pm 0.7 \mathrm{~mm}$ at the midpoint of the MPFL origin and $7.1 \pm 0.9 \mathrm{~mm}$ at the distal origin. Thus MPFL took origin from the anterior $18-44 \%$ of anteroposterior patellar depth, extending over $26 \%$ of the depth of the medial border of the patella (Fig. 2).

\section{The course and insertion of the deep MPFL ligament}

In all 30 specimens the MPFL ligament was present. We found the MPFL to have superficial and deep parts. 


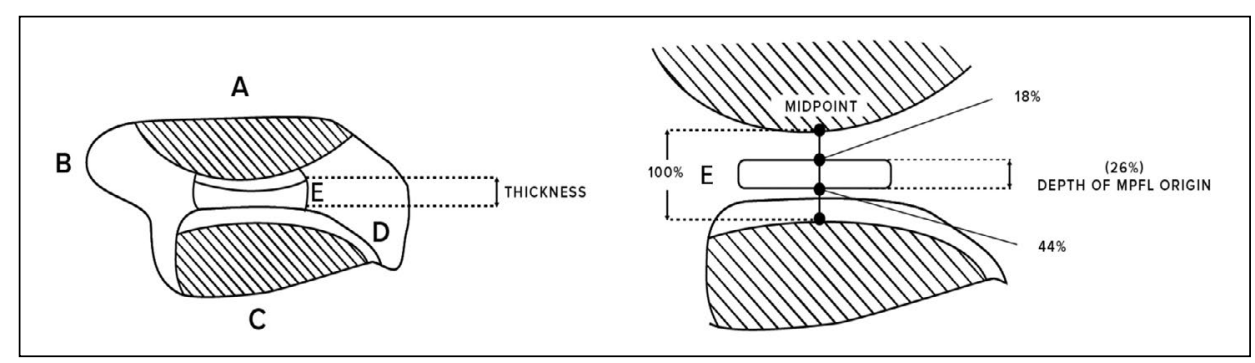

Figure 2. Dimensions of medial patellofemoral ligament (MPFL) origin from medial patella; $A$ — anterior border of patella; $B$ - inferior margin of patella; $\mathrm{C}$ - articular surface of patella; D - edge of cartilage; $\mathrm{E}$ - origin of MPFL.

The deep MPFL (dMPFL) was the more substantial part of the ligament. Its origin was from the suprapatellar tendon, the patella and the infrapatellar ligament as described above (Fig. 3). It ran medially to the insertion onto the distal femur.

There were three patterns of dMPFL insertion to the femur (Fig. 4):

- Type 1: in 22/30 (73\%) specimens dMPFL inserted into the adductor tubercle by narrow, thin fibre bundles (Fig. 5A).

- Type 2: in 5/30 (17\%) of the knee specimens, both the superficial MPFL (sMPFL) and the dMPFL bifurcated around the medial epicondyle. The dMPFL distal bundle was relatively thin and inserted into the superficial medial collateral ligament. The dMPFL proximal bundle was relatively wide and inserted into the adductor tubercle (Fig. 5B).

- Type 3: in 3/30 (10\%) of specimens, a widened dMPFL inserted into the proximal part of superficial medial collateral ligament and on to the medial epicondyle and adductor tubercle (Fig. 5C).

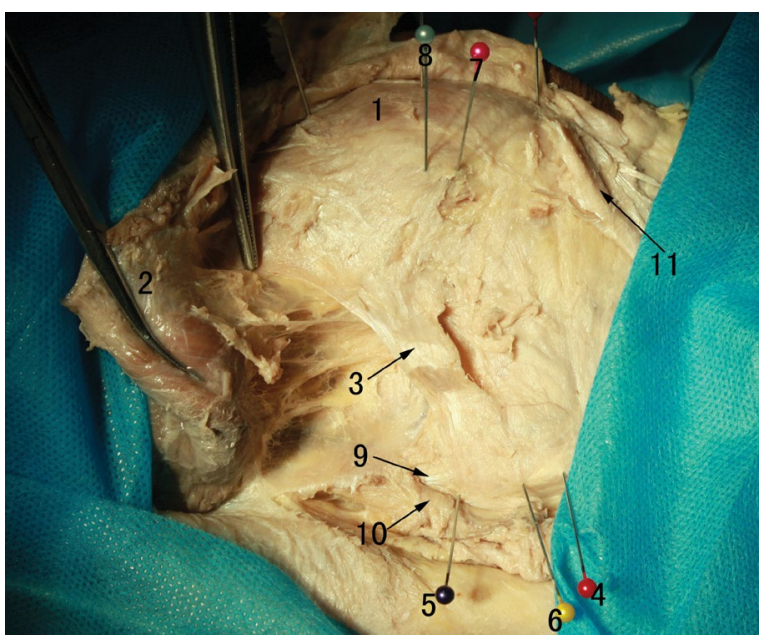

Figure 3. The deep medial patellofemoral ligament; 1 - patella; 2 - vastus medialis oblique; 3 - deep medial patellofemoral ligament (dMPFL); 4 - medial epicondyle of femur; 5 - superior border of dMPFL femoral attachment; 6 - inferior border of dMPFL femoral attachment; 7 - inferior border of dMPFL patellar insertion; 8 - the convex vertex of patellar medial margin; 9 - great adductor muscle tendon; 10 - adductor muscle tendon sheath; 11 - medial patellotibial ligament.
TYPE 1

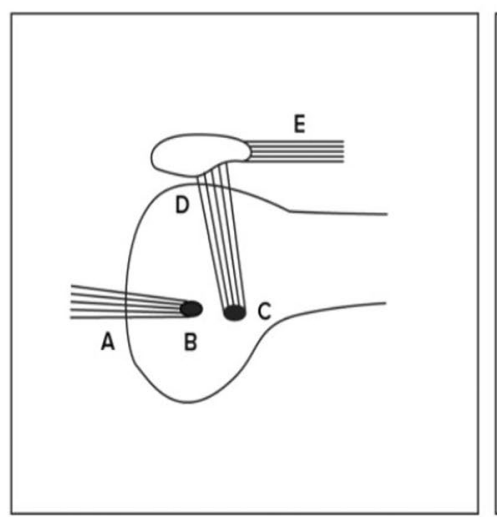

TYPE 2

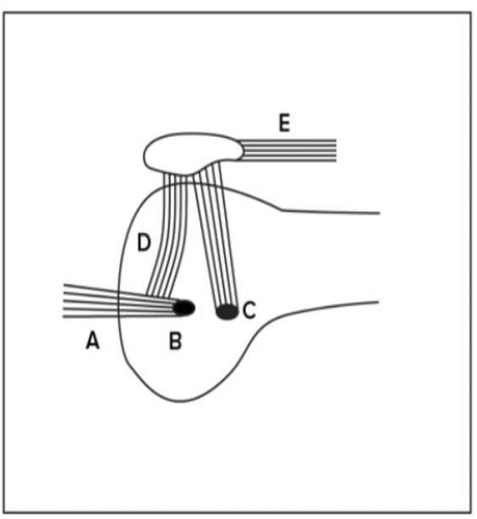

TYPE 3

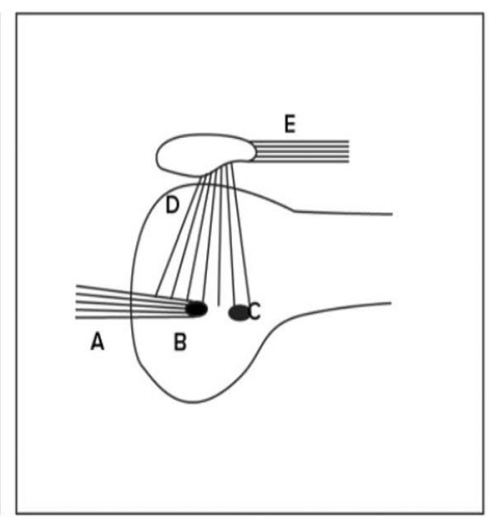

Figure 4. The three insertions of deep medial patellofemoral ligament (MPFL) onto the medial femur; $A$ - medial collateral ligament; B — medial femoral condyle; C — adductor tubercle; D - MPFL; E — suprapatellar ligament. 


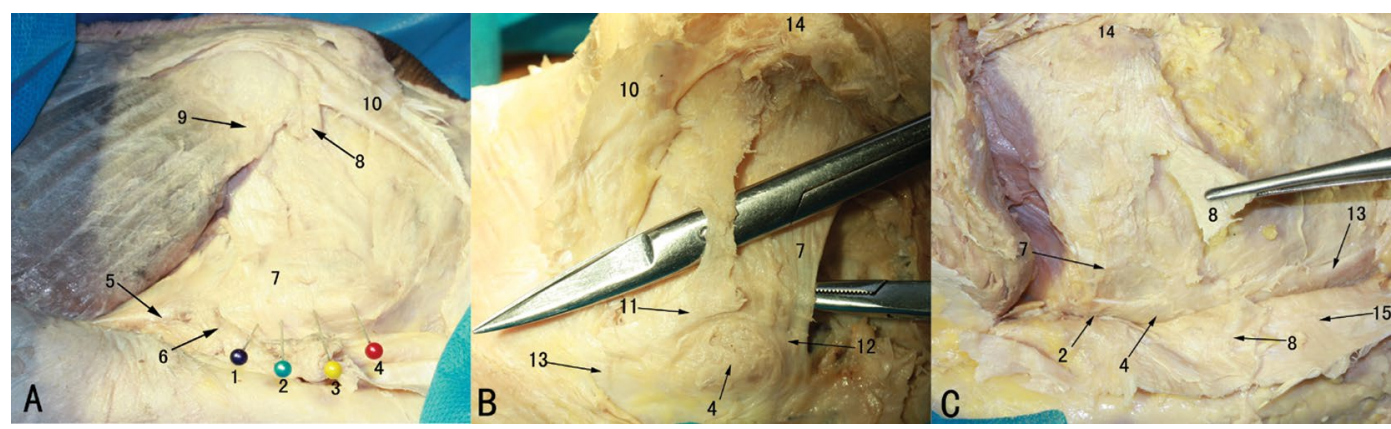

Figure 5. Deep medial patellofemoral ligament (dMPFL) femoral attachment; A. Type 1; B. Type 2; C. Type 3; 1 - margo superior of dMPFL femoral origin point; 2 - adductor tubercle; 3 - margo inferior of dMPFL femoral origin point; 4 - medial epicondyle of femur; 5 - great adductor muscle tendon; 6 - great adductor muscle tendon sheath; 7 -dMPFL; 8 - superficial MPFL (cut off); 9 - vastus medialis oblique aponeurosis; 10 - medial patellotibial ligament; 11 - anterior bundle of superficial MPFL; 12 — posterior bundle of dMPFL; 13 - the superficial medial collateral ligament; 14 — patella; 15 — superficial medial patellar retinaculum.

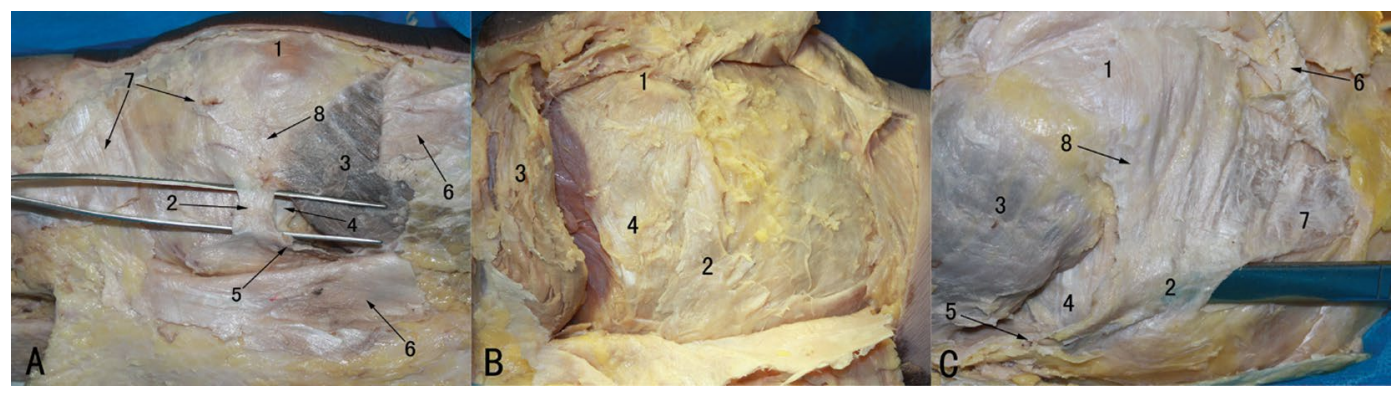

Figure 6. Deep medial patellofemoral ligament (MPFL) and superficial MPFL; A. Type1; B. Type 2; C. Type 3; 1 - patella; 2 - superficial MPFL; 3 - vastus medialis oblique; 4 - deep MPFL; 5 - adductor magnus tendinous sheath and its extension; 6 - superficial medial patellar retinaculum; 7 - medial patellotibial ligament; 8 - aponeurosis of vastus medialis oblique.

Table 1. Measurements of the deep medial patellofemoral ligament (MPFL) insertion onto the femur

\begin{tabular}{lcc}
\hline & $\begin{array}{c}\text { Distance between the midpoint } \\
\text { of the femoral footprint of MPFL } \\
\text { and adductor tubercle [mm] }\end{array}$ & $\begin{array}{c}\text { Width of MPFL } \\
\text { femoral footprint } \\
\text { [mm] }\end{array}$ \\
\hline Type 1 & $1.0 \pm 0.4$ & $11.6 \pm 1.1$ \\
Type 2 & $4.7 \pm 1.6$ & $13.4 \pm 1.0$ \\
Type 3 & $9.5 \pm 0.9$ & $27.0 \pm 1.1$ \\
Average & $2.5 \pm 2.9$ & $13.4 \pm 4.8$ \\
\hline
\end{tabular}

The measurements of the insertion into the adductor tubercle for types 1, 2 and 3 are given in Table 1.

\section{The anatomy of the superficial MPFL}

There were three patterns of origin and substance of the much smaller sMPFL:

- Type 1: (27/30: 90\% specimens). The sMPFL arose from the confluence of the vastus medialis oblique aponeurosis and the medial patellotibial ligament.
It then ran along the distal edge of the dMPFL. On the deep surface of the sMPFL there were delicate fibre connections with the dMPFL (Fig. 6A).

- Type 2: (2/30: 7\% specimens). The sMPFL arose from the medial border of the patella distal to the origin of dMPFL. It then ran parallel and distal to the dMPFL (Fig. 6B).

- Type 3: (1/30: 3\% specimens). The sMPFL was wide and substantial. This type arose from the confluence of the vastus medialis oblique aponeurosis and the medial patellotibial ligament like type 1, but was comparatively more dense and wider. There were no fibre connections with the dMPFL in this type (Fig. 6C).

All three types of sMPFL inserted into the adductor magnus tendinous sheath and its extension to the tibia.

\section{DISCUSSION}

\section{The MPFL origin from the patella}

We found the medial origin of the MPFL to be the distal suprapatellar tendon, the medial surface of 
the patella and the proximal part of the infrapatellar ligament.

The literature describes a variety of patellar origins of the MPFL. According to Aragao et al. [2], in 40\% of specimens MPFL was attached to more than 2/3 of the whole medial margin of the patella, and in $27 \%$ was attached to the whole medial margin of the patella. Steensen et al. [17] demonstrated that the lateral width of the MPFL origin from the patella was $38.8 \%$ of the entire patellar height. Tanaka et al. [19] found MPFL present in 28 of 32 knees, and in one specimen the MPFL was attached to the entire medial surface of the patella. In another the MPFL took origin from the quadriceps tendon alone. Tanaka et al. [18] described the intersection of the medial border of vastus intermedius tendon and the articular surface of the patella as the approximate midpoint of the MPFL complex.

\section{The substance of the MPFL}

We found the MPFL to consist of superficial and deep parts, with three patterns of anatomy of the substance of the MPFL.

Warren and Marshall [21], described the MPFL between the superficial fascia and the joint capsule, connected to isolated transverse fibrous tissue between the attachment of superficial medial collateral ligament and the medial edge of the patella.

\section{The MPFL insertion to the femur}

We found that in $70 \%$ of specimens the MPFL inserted solely into the adductor tubercle. Two other patterns involving insertion into the medial epicondyle and MCL were also found.

Smirk and Morris [16] believed the MPFL originated from $1 \mathrm{~cm}$ away from the distal of the adductor tubercle posterior to femur medial epicondyle, ended at the inner upper edge of the patella, the lower surface of vastus medialis oblique and the quadriceps muscle. Yu et al. [22] found by dissection, that the MPFL originated from femoral adductor tubercle and formed a fan-shaped divergence forward, the upper part of the fibres were in the deep of VMO, went upward and forward to fuse with the vastus intermedius muscle fascia, the central fibres went forward to fuse with the VMO aponeurosis and ended in the superior of the medial border of the patella. The lower fibres went forward and downward, ended in the superior and middle of the medial margin of the patella. According to Avikainen et al. [3] and Hautamaa et al. [9], the MPFL was located in the insertion of the femoral medial epicondyle. According to Feller et al. [8], it was in front of the femoral medial epicondyle. Conlan et al. [5] believed it was in the adductor tubercle. The anatomical study by Tuxoe et al. [20] found that it was at the femoral attachment point close to the medial collateral ligament distal from the adductor tubercle. Nomura et al. [13], Panagiotopoulos et al. [14], and Aragao et al. [2] believed the normal MPFL started between the femoral medial epicondyle and adductor tubercle.

\section{CONCLUSIONS}

We have described the anatomy of the MPFL in detail. This ought to help guide both the interpretation of MRI scans of the MPFL and operative procedures to reconstruct the MPFL.

Regarding placement of the patellar tunnel: If a single tunnel is used this should be placed at the junction of the proximal $1 / 3$ and distal 2/3 of vertical patellar height (and at the most medial part of the medial border of the patella). In the anteroposterior diameter the tunnel should be placed at the junction of the anterior $1 / 3$ and posterior $2 / 3$ of the depth of the patella. If two tunnels are used, they should be superior and inferior to this point and centred on it.

The femoral tunnel should be centred $2.5 \mathrm{~mm}$ distal to the adductor tubercle.

\section{Acknowledgements}

The authors gratefully acknowledge the assistance of Prof. Yu Jun Xia and Prof. Zeng Tao Wang in providing the specimens and other experiment materials. This work was supported by Scientific Research Support Fund for Teachers of Jining Medical University (Grant No.JY2017FS027). Christopher Ricketts provided the illustrations.

\section{REFERENCES}

1. Amis AA, Firer P, Mountney J, et al. Anatomy and biomechanics of the medial patellofemoral ligament. Knee. 2003; 10(3): 215-220, indexed in Pubmed: 12893142.

2. Aragão JA, Reis $F P$, de Vasconcelos DP, et al. Metric measurements and attachment levels of the medial patellofemoral ligament: an anatomical study in cadavers. Clinics (Sao Paulo). 2008; 63(4): 541-544, indexed in Pubmed: 18719768.

3. Avikainen V, Nikku R, Seppänenlehmonen T. Adductor magnus tenodesis for patellar dislocation. Clin Orthopaedics Related Res. 1993; \&NA;(297): 12???16, doi: 10.1097/00003086-199312000-00004. 
4. Burks R, Luker M. Medial patellofemoral ligament reconstruction. Techniques Orthopaedics. 1997; 12(3): 185-191, doi: 10.1097/00013611-199709000-00008.

5. Conlan T, Garth WP, Lemons JE. Evaluation of the medial soft-tissue restraints of the extensor mechanism of the knee. J Bone Joint Surg. 1993; 75(5): 682-693, doi: 10.2106/00004623-199305000-00007.

6. Drez D, Edwards TB, Williams CS. Results of medial patellofemoral ligament reconstruction in the treatment of patellar dislocation. Arthroscopy. 2001; 17(3): 298-306, doi: 10.1053/jars.2001.21490, indexed in Pubmed: 11239352.

7. Du H, Tian XX, Guo FQ, et al. Evaluation of different surgical methods in treating recurrent patella dislocation after three-dimensional reconstruction. Int Orthop. 2017; 41(12): 2517-2524, doi: 10.1007/s00264-017-3552-9, indexed in Pubmed: 28702749.

8. Feller JA, Feagin JA, Garrett WE. The medial patellofemoral ligament revisited: an anatomical study. Knee Surgery, Sports Traumatology, Arthroscopy. 1993; 1(3-4): 184-186, doi: 10.1007/bf01560202.

9. Hautamaa PV, Fithian DC, Kaufman KR, et al. Medial soft tissue restraints in lateral patellar instability and repair. Clin Orthop Relat Res. 1998(349): 174-182, indexed in Pubmed: 9584380.

10. Kaplan EB. Factors responsible for the stability of the knee joint. Bull Hosp Joint Dis. 1957; 18(1): 51-59, indexed in Pubmed: 13510947.

11. Muneta T, Sekiya I, Tsuchiya M, et al. A technique for reconstruction of the medial patellofemoral ligament. Clin Orthop Relat Res. 1999(359): 151-155, indexed in Pubmed: 10078138.

12. Nomura E, Horiuchi Y, Kihara M. A mid-term follow-up of medial patellofemoral ligament reconstruction using an artificial ligament for recurrent patellar dislocation. The Knee. 2000; 7(4): 211-215, doi: 10.1016/s09680160(00)00072-7.

13. Nomura $E$, Inoue $M$, Osada N. Anatomical analysis of the medial patellofemoral ligament of the knee, especially the femoral attachment. Knee Surg Sports Traumatol
Arthrosc. 2005; 13(7): 510-515, doi: 10.1007/s00167004-0607-4, indexed in Pubmed: 15895206.

14. Panagiotopoulos E, Strzelczyk P, Herrmann M, et al. Cadaveric study on static medial patellar stabilizers: the dynamizing role of the vastus medialis obliquus on medial patellofemoral ligament. Knee Surg Sports Traumatol Arthrosc. 2006; 14(1): 7-12, doi: 10.1007/s00167-0050631-z, indexed in Pubmed: 16001289.

15. Sanders TG, Morrison WB, Singleton BA, et al. Medial patellofemoral ligament injury following acute transient dislocation of the patella: MR findings with surgical correlation in 14 patients. J Comput Assist Tomogr. 2001; 25(6): 957-962, indexed in Pubmed: 11711811.

16. Smirk $\mathrm{C}$, Morris $\mathrm{H}$. The anatomy and reconstruction of the medial patellofemoral ligament. Knee. 2003; 10(3): 221-227, indexed in Pubmed: 12893143.

17. Steensen RN, Dopirak RM, McDonald WG. The anatomy and isometry of the medial patellofemoral ligament: implications for reconstruction. Am J Sports Med. 2004; 32(6): 1509-1513, doi: 10.1177/0363546503261505, indexed in Pubmed: 15310579.

18. Tanaka MJ, Voss A, Fulkerson JP. The Anatomic Midpoint of the Attachment of the Medial Patellofemoral Complex. J Bone Joint Surg Am. 2016; 98(14): 1199-1205, doi: 10.2106/JBJS.15.01182, indexed in Pubmed: 27440568.

19. Tanaka MJ. Variability in the Patellar Attachment of the Medial Patellofemoral Ligament. Arthroscopy. 2016; 32(8): 1667-1670, doi: 10.1016/j.arthro.2016.01.046, indexed in Pubmed: 27132768.

20. Tuxøe JI, Teir M, Winge S, et al. The medial patellofemoral ligament: a dissection study. Knee Surg Sports Traumatol Arthrosc. 2002; 10(3): 138-140, doi: 10.1007/s00167001-0261-z, indexed in Pubmed: 12012030.

21. Warren LF, Marshall JL. The supporting structures and layers on the medial side of the knee. J Bone Joint Surg. 1979; 61(1): 56-62, doi: 10.2106/00004623-197961010-00011.

22. Yu CS, Xuan Y, Li KC. Anatomic study of the medial patellar retinaculum and its clinical implication. Chinese J Clin Anat. 2004; 22(3): 263-265. 